\title{
Cambios en el abordaje del turismo ${ }^{1}$
}

\author{
DANIEL HIERNAUX-NICOLAS
}

Universidad Autónoma de Querétaro, Santiago de Querétaro, México

\section{La concepción tradicional de turismo frente a las nuevas prácticas turísticas}

¿Cuáles fueron los principales cambios en la definición y el abordaje del turismo?

Las definiciones del turismo fueron formándose a lo largo del desarrollo de la actividad y bajo dos perspectivas: la primera, tener un referente susceptible de ser aplicado en las estadísticas de todos los países bajo la férula de la Organización Mundial del Turismo (OMT). La segunda, desde diversas áreas del conocimiento que se interesaban en el turismo, el derecho y la economía principalmente. Eso no quiere decir que se aplicó uniformemente en todo el mundo. En México, por ejemplo, los cruces de turistas estadounidenses hacia las localidades fronterizas del norte de ese país, no se tomaron en cuenta como viajes turísticos durante muchos años. Fue necesario que, un momento dado, las autoridades rectificaran los datos- obviamente, solo contando a quienes pernoctaban-. También vale señalar que el mandamiento estricto de que "turista es quien viaja por razones de ocio únicamente", fue borrado de la definición de la OMT. De esta manera, el turismo en el sentido tradicional y el viaje de negocios -con eventualmente la toma de unos días libres para visitar la ciudad o la región de destino-, se han mezclado en las estadísticas. Eso ha permitido, entre otros factores, que las cantidades de turistas internacionales fueran registradas como crecientes a lo largo de sesenta años, salvo en escasos momentos de fuerte crisis como el ataque a las Torres Gemelas de Nueva York, en 200 .

Por otra parte, a medida que no solo las ciencias administrativas sino también las Ciencias Sociales desarrollaron estudios sobre turismo, fue necesario afinar las definiciones, reflexionar sobre la diferencia real entre turistas y excursionistas (más allá del hecho tangible de pernoctar o no),

1 Entrevista realizada al Dr. Daniel Hiernaux-Nicolas por parte de la Dra. Mariela Demarchi, docente del Departamento de Geografía (FHUC-UNL). 
en fin, repensar qué es turismo y qué es actividad de diversión para una población metropolitana o regional. Remito, por ejemplo, a los trabajos de David Crouch al respecto, que aboga por la desaparición de la diferencia entre ocio y turismo.

¿Es posible pensar estos cambios desde el paso del tradicional turismo fordista a las nuevas prácticas posfordistas? ¿Y desde la ruptura -o node la dicotomía ocio-trabajo?

Ambas partes de su pregunta son muy oportunas: el cambio en las definiciones del turismo depende también del modelo o de los modelos de turismo en actividad, en un momento dado. Ciertamente, las nuevas prácticas de turismo, sean llamadas posfordistas o no, imponen reconsiderar las definiciones en torno a él. Personalmente, sigo creyendo que es prácticamente imposible medir los flujos de turismo, en especial los de turismo doméstico (interno a los países). Les doy un ejemplo: durante muchos años trabajé sobre las periferias recientes y muy pobres de la ciudad de México. Pues resultó que aun en esos estratos sociales que rayan en la mera subsistencia, se puede encontrar familias que "se toman vacaciones". La explicación es simple: siendo muchas veces migrantes de primera o segunda generación, muchos habitantes de las periferias tienen familiares que viven en ciudades o pueblos de provincia y que pueden acogerlos durante días de asueto. Se trata de un turismo familiar, de escaso gasto, pero turismo al fin. A lo que no hay que olvidar de agregar un turismo religioso marcado por peregrinaciones, en las cuales los peregrinos caminan y se alojan en tiendas de campaña o duermen en la calle. ¿Son turistas? En cierta forma, sí, siguiendo las definiciones oficiales. ¿Se sienten turistas? Generalmente no; es algo que hay que analizar, por cierto. La diferencia entre considerarse turista o simple viajero por motivos personales, de salud, religiosos, etc.

Por otra parte, la ruptura de la dicotomía entre turismo y ocio que señalaba al final de mi primera respuesta, va en el mismo sentido de la necesidad de volver a barajar los naipes. ¿Quién es quien? ¿Quién es turista? ¿Quién no? Inclusive, y eso tiene un carácter paradójico, he podido oír en entrevistas a turistas estadounidenses en México defenderse de ser "an ugly American tourist" refiriéndose al cliché bien real del "gringo" con gorras de béisbol, pantalón corto, sandalias con calcetines y camisa hawaiana. Los que los denuncian se presentan como 
turistas respetuosos, en busca de cultura, de tradiciones, etc. Un tema para reflexionar, ¿verdad?

Recuerden también esa entrevista a Zygmunt Bauman, cuando planteaba que el turista es la metáfora del hombre global actual. Todos somos un poco turistas en muchos momentos de nuestras vidas y, en ocasiones, somos turistas sin quererlo al realizar actividades consideradas tradicionalmente como "turísticas". Todo eso nos plantea algo que creo que es fundamental: dejemos de lado esas interpretaciones tradicionales, es hora de estudiar con la mente libre y dispuesta a analizar las prácticas y escuchar las narrativas... después pensaremos en qué es qué y quién es quien...

\section{La participación de la Geografía en los debates sobre la práctica turística}

¿En qué medida influyeron los cambios mencionados en el posicionamiento de la Geografía para el abordaje de los estudios turísticos? ¿Los cambios en las prácticas turísticas le permiten a la Geografía repensar la categoría espacio?

Bueno, esa es una pregunta doble y compleja. La Geografía tradicional del turismo partió de un no tan famoso escrito de Walter Christaller, ese geógrafo nazi reconvertido al comunismo cuando Alemania perdió la guerra, amante del orden y de los modelos. La pregunta de su artículo era buscar entender por qué el turismo no cuadraba en su modelo hexagonal de mercado y lugares centrales. En eso anticipaba bien la forma como la Geografía iba a analizar el turismo en una primera etapa: como un factor de organización del espacio. También podemos encontrar un trabajo de Miossec - texto muy conocido en la Geografía francesa- que muestra claras influencias christallerianas, además de seguir los planteamientos de la Geografía aplicada a la Phliponneau, entre otros.

No pienso que son los cambios que se mencionaron antes los que movieron a la Geografía en su enfoque al estudio del turismo. Más bien, es la dinámica interna de la disciplina la cual se ha puesto más exigente sobre su definición del espacio y se ha alimentado de numerosos debates en las Ciencias Sociales -entre las cuales el espacio era presente, a veces abiertamente a veces de manera soterránea-. ¿¿Recuerdan la entrevista que le hizo Yves Lacoste a Michel Foucault para el primer número de su revista Herodote? Foucault se defendió de un acercamiento a la Geografía hasta que Lacoste le demostró que salvo que se quedara con 
una visión tradicional de una Geografía enumerativa y descriptiva, en realidad hacía Geografía o Sociogeografía, quizás. Pensemos cómo el concepto de poder aplicado al espacio ha sido importante para nosotros, geógrafos, así como los de panóptico y de heterotopía -o mejor dicho de "espacio otro"-.

Por supuesto que, con el paso del tiempo, los geógrafos (sigo la RAE, el masculino genérico representa a hombres y muchas mujeres que han aportado a la Geografía) han revisitado su manera de investigar el turismo, para no quedarse solo en los análisis cuantitativos y la cartografía de datos turísticos, sino en vista a plantear un análisis profundo de un fenómeno centralmente societario.

Que los cambios en las prácticas turísticas le permiten a la Geografía repensar el concepto de espacio, pues creo que es darle demasiada influencia al estudio del turismo y a las prácticas de éste; como si fuera susceptible de modificar algo tan fundamental para la Geografía como el concepto del espacio. La Geografía ha operado esos cambios desde adentro y, con toda seguridad, mano a mano con las Ciencias Sociales. El estudio del turismo, posiblemente, refuerce algunas reflexiones que ya han surgido en la epistemología de la Geografía, esto es muy posible, pero no es el aporte esencial.

El giro cultural en general y el giro espacial en particular han generado nuevas maneras de concebir la Geografía y los campos de estudio. En este sentido: ¿Es posible pensar en nuevos aportes teóricos e instrumentos metodológicos para el estudio turístico?

Los giros que se mencionan en la pregunta han sido decisivos para la recomposición de la Geografía, disciplina que por desgracia no entienden -o no quieren entender- nuestros políticos neoliberales. No diré nombres, saben a quiénes me refiero. Pero, cuidado, aun en países más socialdemócratas como varios europeos, el desprecio a la Geografía es evidente; se ha quitado de las asignaturas de la educacion básica y creo que por dos razones: la primera por la mala fama de la Geografía descriptiva tradicional, la cual se vuelve cada vez más obsoleta e inútil frente al avance de las tecnologías de manejo y representación de datos. Esa mala fama se ganó a pulso, la verdad. Pero también hay otra faceta que a veces ignoramos o escondemos, pero que es esencial: la Geografía es un arma que puede servir para desmontar y enfrentar narrativas neoli- 
berales. Piensen, por ejemplo, en la expansión de la desforestación o de la minería en América Latina: nada como un buen mapa para demostrar la expoliación, la destrucción ambiental y otros efectos sobre las poblaciones locales. En ese sentido, la Geografía es peligrosa.

Yo siento que el potencial de la Geografía "revisitada" por los giros es enorme. Creo, inclusive, que los geógrafos no nos damos cuenta de que lo que tenemos en mano, es un potencial insospechado, realmente impresionante, para ayudar a repensar el mundo y, quizás, en mejorarlo. Con todo lo que hemos aprendido de las Ciencias Sociales y todo lo que podemos o hemos elaborado ya desde adentro de la Geografía, tenemos un potencial conceptual y metodológico enorme. Milton Santos, tan temprano como en el año 1968 de sentida memoria, llamaba a retomar lo necesario de las Ciencias Sociales, tanto en lo conceptual como en lo metodológico, sin tapujos ni restricción alguna. Hemos tardado en hacerlo, pero lo hacemos, a nuestro ritmo; las resistencias internas son aun sustanciales.

Y claro que, para los estudios turísticos desde la Geografía, el potencial es sustancial. Ya terminemos con esos estudios de consultoría o de instancias de gobierno, con encuestas en aeropuertos, de diez a quince preguntas, conteos de cuartos de hoteles, etc., como técnicas esenciales para los estudios turísticos. Se abre un panorama grande, no una caja de Pandora, sino realmente una caja de herramientas muy valiosas. Pienso en el análisis de las narrativas de los blogs de viaje, el recurso al análisis del discurso de las guías de viaje o de las páginas de compra en línea (piensen por ejemplo en esa tendencia de AirBnB en hablar del viaje como "experiencia"), la posibilidad de hacer relatos o mismo historias de vida, claro, no en un aeropuerto sino en otro contexto. Inclusive usar las geolocalizaciones de fotografías subidas, por ejemplo, a Instagram, para determinar el distrito turístico recorrido por los turistas en alguna ciudad; sacar jugo a los Bigdata en turismo, en fin, una multiplicidad de técnicas, mecanismos, instrumentos a nuestra disposición si asumimos que el turismo es un fenómeno societario, que se puede analizar desde una ciencia socioespacial, que es la Geografía. Y desde ahora, podemos decir que se usan esos instrumentos y se han hecho estudios de ese corte, pero falta mucho por inventar y muchas áreas que cubrir.

Lo anterior, para responder a los aportes metodológicos. En referencia a los teóricos, pues, no hay duda de que la nueva forma de entender y 
pensar el espacio, que surge con la Geografía actual, permite replantear muchos aspectos del turismo. Un solo ejemplo: las Ciencias Sociales y las Humanidades nos han hecho ver que se han acrecentado el carácter efímero de nuestros modos de vida y nuestras relaciones interpersonales, entre otros. El turismo es efímero, aun cuando el capital haya querido volverlo duradero, anclado en el territorio con objetos físico-materiales. Pensemos, por ejemplo, qué puede significar al largo plazo -éste nuevo imaginario sobre los desplazamientos en avión-. Los suecos empezaron ya una gran transformación por la cual prefieren viajar más horas y de manera menos confortable por tierra que por aire, por la huella ecológica del desplazamiento por vía aérea ¿Cómo se comportará el turismo en unos quince ańos, si ese imaginario prospera? ¿Qué pasará con los destinos distantes -pienso en el fin de América del Sur, como Ushuaia, por ejemplo, si se generaliza la tendencia de no tomar el avión para las vacaciones? Es un reto geográfico con toda seguridad, pero también un inmenso problema social. Ya tenemos sitios degradados, que fueron exitosos en tiempos pasados, por ejemplo, algunos lugares termales de México, que se están volviendo quizás las primeras ruinas del modelo turístico antiguo ¿Quizás, un día, los aeropuertos estarán vacíos?

Por otra parte, las vacaciones son ahora una acumulación (en cantidad y distancia/recorrido acorde con la cartera de cada viajero) de destinos para microestancias: lo efímero se impone...

Desde una Geografía constructivista y considerando las prácticas -materiales y no materiales- individuales: ¿Cómo es posible abordar ciertas problemáticas turísticas?

En cierta medida, ya contesté esa pregunta en el sentido de que todos los giros en la Geografía actual son puertas abiertas para quien quiere avanzar en las metodologías. Las prácticas individuales en el turismo, es evidente que son una mina de información relativamente virgen. ¿Por qué ese abandono? Justamente, por la manera como se definió el turismo y se integró un modelo estadístico que parecía la única forma de analizarlo. Sin embargo, es justo reconocer que los psicólogos, por una parte, pero también las personas que hacen estudios de mercado, por la otra, han abundado en el estudio de las prácticas, aunque en lo general, para alimentar estudios formales a vocación empresarial. 
Debemos ampliar ese campo, porque el turismo se está redefiniendo a partir de las prácticas individuales y los imaginarios que las conducen o que se derivan de esas experiencias. Finalmente, me atravería a decir que el turismo no es más que sus prácticas. Si no quiero irme de vacaciones, ni toda la publicidad y los descuentos me pueden hacer cambiar de idea. Nuestras prácticas son decisivas para el derrotero que quieren seguir las empresas. Ahora, los grupos hoteleros están muy atentos a la reacción de los turistas en sus compras de viajes y estancias. La penetración de las plataformas "peer to peer" puede cambiar el curso de la historia del turismo, por eso mismo hay tanto odio y presión sobre los gobiernos en contra de las mismas, por parte de las empresas hoteleras. Más que nunca, se evidencia que trabajan en un mercado efímero, que puede modificar sus preferencias.

Regresando a las cuestiones metodológicas, usar cartografía participativa pudiera, por ejemplo, ser una buena forma de hacer narrar sus vacaciones por el turista. Dónde fue, qué vio, qué no vio, lugares que sintió atractivos y otros peligrosos, etc. Más que una charla, un buen mapa podría ser útil. Ahora bien, el turista es justamente un ser volátil, por lo que habrá que pensar cómo captarlo, dónde, en qué horario, etc. Sin embargo, no debería ser más complicado que entrevistar a un trabajador de una planta industrial, un estudiante o un vendedor de tienda departamental. Cada uno tiene sus restricciones, lugares donde conviene o no entrevistarlos o hacerles participar de un proceso investigativo como la cartografía participativa; habrá que desplegar una fuerte inteligencia en la selección y puesta en práctica de instrumentos para lograr buenos resultados. Son solo ejemplos, quizás sería bueno realizar seminarios de discusión para definir esas maneras de aplicar métodos asociados a la Geografía constructivista al estudio de ese proceso tan específico que es el turismo.

\section{La Geografía del turismo en América latina}

¿Qué lugar ocupa la Geografía latinoamericana en los estudios turísticos? ¿En qué medida la Geografía cumple un rol como disciplina en la gestión y el ordenamiento territorial de los destinos turísticos en América latina?

La Geografía sí ocupa un rol importante en los estudios turísticos. Revisando algunos casos de memorias de congresos de estudios turísticos, 
es notorio que ha crecido la cantidad de estudios de corte geográfico, aunque no forzosamente realizados por geógrafos. Se debe en buena medida a que el turismo es un proceso eminentemente espacial, como lo he afirmado en repetidas ocasiones. Su esencia es el viaje, el desplazamiento, y por ende, esta dimensión espacial se hace presente en los estudios. No -o no solo- como mera localización, sino como componente intrínseca al proceso turístico. Pensemos, por ejemplo, en lo que se llama el "turismo residencial" o "turismo de segundas residencias" ¿Cómo es la huella espacial de ese tipo de "residencia"? ¿Cómo podría ser posible eludir la dimensión espacial? La misma conduce a los investigadores, sean sociólogos, historiadores, o lo que sea, a desarrollar una dimensión espacial a su trabajo, y eso de manera totalmente natural. Los invito a revisar los estudios publicados, por ejemplo, por el grupo de investigadores de la Universidad de Alicante; el hecho de tomar en cuenta el espacio es totalmente natural para los investigadores.

Podemos decir en cierta forma, que es una suerte de maridaje de una cepa geográfica con otras de Ciencias Sociales... ¿Qué no son esos los mejores vinos?

La segunda parte de la pregunta es obvia: el ordenamiento territorial de los destinos turísticos descansa mucho en la disciplina geográfica, aunque, como suele ocurrir, son usualmente profesionistas de otras disciplinas que realizan los estudios en consultorías, universidades e instancias de gobierno. Pero suele ocurrir también que "les falta Geografía” y retoman solo lo formal del ordenamiento (claro que es de origen geográfico en general) y los geógrafos deben pelear los espacios donde se producen los estudios de ordenamiento.

\section{¿Los geógrafos investigadores y las universidades ocupan un lugar en particular?}

La pregunta debe contestarse por una respuesta contundente: deben ocupar un lugar no solo particular sino central; diría que preponderante, por ese carácter transversal de la Geografía. No se trata de decir que los geógrafos somos mejores que los demás, posiblemente nuestros conocimientos específicos en tal o cual materia es inferior a las demás disciplinas, pero la visión global, transversal y espacial es indudablemente un plus que debe valorarse. 
Hablando de las universidades, la situación es más difícil por varios motivos. En primer lugar, cuando las universidades sostienen carreras de turismo, esas han dificilmente escapado a la dominancia de lineas educativas de gestión turística. Su capacidad para emprender estudios del turismo que integren también las Ciencias Sociales sin olvidarse de las ciencias "duras" cuando sean necesarias y con toda seguridad las ambientales, suele ser reducida. Solo universidades con perfiles consolidados de larga data en turismo y con apertura intelectual y una buena dosis de dinamismo, son capaces de realizar una apertura en ese sentido. Para el caso mexicano, es notorio que las universidades que iniciaron la enseñanza del turismo, desde hace varias décadas, han emprendido ya un proceso de apertura que permite la eclosión de nuevos perfiles de egresados y el desarrollo de una capacidad para realizar estudios transversales en turismo. Algunas otras, en ocasiones, se inician directamente en esa vía (eluden de plano la dimension de la gestión turística), y al inverso, otras refuerzan lo último considerando que es mejor especializarse en gestión más que recorrer senderos resbalosos y peligrosos de estudios turísticos abiertos a varias disciplinas (es particularmente cierto para las universidades privadas).

\section{¿Cuáles son -o deberían ser- los principales tópicos en la agenda de investigación latinoamericana de la Geografía en relación con los estu- dios turísticos?}

Quizás les parecería que me contradigo, pero no creo que sea así: necesitamos más estudios de localización y estimaciones cifradas de los procesos turísticos. Por ejemplo, en México, quince años después del primer estudio oficial sobre el turismo de segundas residencias, el gobierno no se ha tomado la pena de introducir preguntas en los censos para estimar el parque de viviendas destinadas a ese uso, mientras que numerosos países como España o Francia, tienen no solamente estimaciones detalladas sino además, una cartografía excelente y envidiable que aclara y refleja el proceso de crecimiento de la vivienda para el turismo de segundas residencias. Me parece fundamental que se mejore el conocimiento cuantitativo y espacial del turismo, como punto de partida de otros estudios.

Obviamente, abogo por los estudios cualitativos de todos tipos, en primer lugar, sobre imaginarios del turismo, que considero esenciales 
para entender las dinámicas del proceso turístico, las tendencias previsibles y demás.

Estudios sobre las reacciones de los residentes frente a esos procesos son necesarios. En muchos países latinoamericanos seguimos viendo el turista como bienvenido. Cuando se masifica la demanda, las opiniones varían. No hablo de impactos: hablar de impactos es un error ya que es considerar el turismo como un fenómeno externo que tiene efectos sobre "algo" extraño al turismo. Todo lo contrario, cuando se observa el turismo urbano, por ejemplo, es evidente que el residente es parte del proceso turístico, sea que vive en un espacio turistificado (quizás en contra de su voluntad) sea que, por ejemplo, los precios de las viviendas y de los bienes en general han crecido por la llegada de turistas. No es un impacto de una suerte de "alien", es una realidad en la cual el residente está inmerso volens nolens.

Estudios de dinámicas sociales, entre la población residente y el turismo: partiendo de lo que acabo de decir, articular el conocimiento de lo que hace, dice, piensa y siente el turista que pudieramos llamar "activo" (el que quiere ser turista) con lo mismo de parte del "turista pasivo" (el que se vuelve involucrado sin querer, el residente).

También los estudios sobre el "habitar" que han avanzado de manera importante no solo desde la filosofía donde anidaban tradicionalmente, sino a la vez, desde las Ciencias Sociales y la Geografía en particular, se han vuelto una prioridad. Entender que el turismo es una forma de "habitar", muy peculiar ciertamente, pero que deja una huella en el territorio, se lo apropia, lo transforma, lo modela a su antojo...

Finalmente, siento la urgencia de una reflexión y debates sólidos sobre lo que queremos del turismo. Ya llegamos a una etapa en la cual el viaje turístico tradicional no es forzosamente una reposición de nuestra fuerza de trabajo, sino una forma de acumular nuevas deudas, de generar estatus y glamur, una manera de consumir que, además, transforma el mundo entero, destruyendo sus culturas locales en la medida que lo volvemos terreno de juego para turistas. Se ha evidenciado, en los últimos años en qué grado esas aparentes nimiedades individuales son, al fin de cuentas, terriblemente degradantes para nuestro planeta y para nuestros grupos humanos que amenazan por la contaminación mercantilista. No se trata ciertamente de cancelar el turismo, ni de reducirlo a operar solo para quienes pueden pagarse un desplazamiento y una estancia de paz, tran- 
quilidad y protección al ambiente, todo envuelto en un lujo superlativo. Se trata de reflexionar sobre un invento relativamente reciente de la humanidad que merece evaluarse con seriedad. Frente a esa tarea, los geógrafos decimos "presentes".

Daniel Hiernaux-Nicolas Querétaro, a Io de junio de 2018

Dedico estas reflexiones a mi nieto Oliver, quien vivirá un futuro tan distinto al que sońaba para mí y para el planeta. 